Real Analysis Exchange

Vol. 24(2), 1998/9, pp. 859-866

Tamás Keleti* Department of Analysis, Eötvös Loránd University, Rakoczi ut 5, 1088 Budapest, Hungary; e-mail: elek@cs.elte.hu

\title{
THE DYNKIN SYSTEM GENERATED BY THE LARGE BALLS OF $\mathbb{R}^{n}$
}

\begin{abstract}
We prove that in an at least three dimensional Euclidean space the Dynkin system generated by the family of all open balls with radii at least one (that is, the smallest collection containing the open balls with radii at least one and closed under complements and countable disjoint unions) does not contain all Borel sets. We also give a simple characterization of the sets of this Dynkin system.
\end{abstract}

A non-empty family $\mathcal{D}$ of subsets of a set $X$ is said to be a Dynkin system provided $\mathcal{D}$ is closed under complements and countable disjoint unions. (The terms ' $\sigma$-class', 'concrete quantum logic' and ' $q$ - $\sigma$-algebra' is also used for the same notion; the latter two in the study of quantum logic.)

In 1988 and 1995 Olejček $[5,6]$ proved that in $\mathbb{R}^{2}$ and in $\mathbb{R}^{3}$ the Dynkin system generated by all balls is equal to the Borel class. (Although it does not make much difference, by a ball we always mean here an open ball.) Recently Jackson and Mauldin [1] and independently Zelený [8] generalized this result for any finite dimensional Euclidean space (in fact, in [1] for any finite dimensional Banach space). In this note we show that in Euclidean spaces with dimension at least three it is not enough using large balls. More precisely we show

Theorem 1. In $\mathbb{R}^{n}$ (for $n \geq 3$ ) the Dynkin system generated by the family of all (open) balls with radii at least 1 does not contain all Borel sets.

The motivation of this result came from infinite dimension: According to two results about the positivity principle for large and small balls in a separable Hilbert space ([3] Theorem 3 and Theorem 4; the proof of the main statement is in [7]; see also in [4]) there is a strange analogy between the small balls in an

\footnotetext{
Key Words: balls, Borel sets, countable disjoint union, complement

Mathematical Reviews subject classification: 28A05, 04A15, 03E15

Received by the editors September 18, 1998

* This research was done when the author visited the University College London having a Royal Society/NATO Postdoctoral Fellowship Award.
} 
infinite dimensional Hilbert space and the large balls in the finite dimensional one. The analogy turned out to be true enough to enable the solution of the following infinite dimensional question, which was asked 20 years ago: "Is it true that in any separable Banach space the Dynkin system generated by the family of all balls is equal to the Borel class?" Generalizing the proof of the above Theorem 1 it is proved in [2] that the answer is negative in an infinite dimensional separable Hilbert space. Actually, the proof in [2] carries over without any essential change to showing Theorem 1 . The aim of this note is to show the simpler original proof of Theorem 1.

We shall also give a complete characterization of the sets of the Dynkin system generalized by the large balls of $\mathbb{R}^{n}(n \geq 3)$.

The idea of the proof of Theorem 1 is to show that the sets from the Dynkin system generated by the large balls, modulo a countable set, can be obtained by a relatively simply operation directly from large balls; in fact, as a union of simple building blocks.

Under a generalized ball in $\mathbb{R}^{n}$ we will understand either a ball, or a halfspace or the whole space $\mathbb{R}^{n}$; by a generalized sphere we will mean a sphere or a hyper-plane. By the radius of an unbounded generalized ball or generalized sphere we will mean infinite.

A stereographic projection $P$ maps the generalized spheres to (normal) spheres on a fixed sphere. We equip the set of generalized spheres by the Hausdorff distance of the mapped spheres; that is,

$$
d(S, T)=\max \left(\sup _{x \in P(S)} d(x, P(T)), \sup _{y \in P(T)} d(y, P(S))\right) .
$$

One can easily check the following:

Lemma 2. Any monotone union or intersection of generalized balls with radii at least 1 is a generalized ball with radius at least 1. Moreover, if the union is not $\mathbb{R}^{n}$ then its boundary belongs to the closure of the set of the boundaries of the generalized balls from $\mathcal{B}$.

If the intersection of different generalized spheres $S, T$ contains more than one point then we shall say that $S, T$ are crossing.

We will use the following obvious fact:

Lemma 3. Let $S \neq T$ be crossing generalized spheres. Then there is $\varepsilon>0$ such that any two generalized spheres $S^{\prime}, T^{\prime}$ with $d\left(S^{\prime}, S\right)<\varepsilon$ and $d\left(T^{\prime}, T\right)<\varepsilon$ are crossing.

The following lemma will ensure that the exceptional set will be countable: 
Lemma 4. Suppose that $\mathcal{S}$ is a family of pairwise non-crossing generalized spheres in $\mathbb{R}^{n}$. Then the set $M$ of those points that belong to at least two different generalized spheres from $\mathcal{S}$ is countable.

Proof. For every $x \in M$ choose generalized spheres $S, T \in \mathcal{S}$ such that $S \neq T$ and $x \in S \cap T$; denote by $U, V, W$ the three components of $\mathbb{R}^{n} \backslash(S \cup T)$, where the notation is such that $\partial U=S, \partial V=T$ and $x$ is the only point of $\bar{U} \cap \bar{V}$, and choose points with rational coordinates $u \in U, v \in V$, and $w \in W$. We claim that $x$ is uniquely determined by the triple $\langle u, v, w\rangle$; since the set of such triples is countable, this will finish the proof. To prove the claim, suppose, that from some $x^{\prime} \in M$ we arrived to the same $\langle u, v, w\rangle$ using generalized spheres $S^{\prime}, T^{\prime} \in \mathcal{S}$ and components $U^{\prime}, V^{\prime}, W^{\prime}$ of $\mathbb{R}^{n} \backslash\left(S^{\prime} \cup T^{\prime}\right)$. Since the generalized spheres from $\mathcal{S}$ are non-crossing and since $U \cap U^{\prime} \neq \emptyset$, we have that $U \subset U^{\prime}$ or $U^{\prime} \subset U$; similarly for $V^{\prime}$ 's and $W$ 's. Exchanging the role of $x$ and $x^{\prime}$ and/or of $U^{\prime}$ 's and $V^{\prime}$ 's if necessary, there are only two cases to consider: (a) $U \supset U^{\prime}$ and $V \supset V^{\prime}$ and (b) $U \subset U^{\prime}, V \supset V^{\prime}$ and $W \subset W^{\prime}$. In case (a) we recall that $\bar{U} \cap \bar{V}$ contains only $x$ and that $x^{\prime} \in \overline{U^{\prime}} \cap \overline{V^{\prime}}$, so $x=x^{\prime}$. In case (b) we get from $W \subset W^{\prime}$ that $U^{\prime} \subset \overline{U \cup V}$; so since $U^{\prime}$ is open and connected, and $\bar{U} \cap \bar{V}$ is just one point, we infer that $U^{\prime}=U$ and we are back in the already proved case (a).

The points $x, y \in \mathbb{R}^{n}$ are said to be separated by a generalized sphere $S$ if they lie in different components of $\mathbb{R}^{n} \backslash S$. For a class of generalized spheres $\mathcal{S}$ let $\sim_{\mathcal{S}}$ be the equivalence relation on $\mathbb{R}^{n} \backslash \bigcup_{S \in \mathcal{S}} S$ defined by

$$
x \sim_{\mathcal{S}} y \quad \leftrightarrow \quad x, y \text { are separated by no generalized spheres of } \mathcal{S} .
$$

Lemma 5. Suppose that $\mathcal{S}$ is a non-empty closed family of pairwise noncrossing generalized spheres with radii at least 1 . Then each equivalence class of $\sim_{\mathcal{S}}$ is of the form $B_{0} \backslash \bigcup_{i>1} \bar{B}_{i}$, where $B_{0}$ is a generalized (open) ball; $B_{1}, B_{2}, \ldots \subset B$ is a (finite or infinite) sequence of pairwise disjoint generalized balls with radii at least 1; and $\partial B_{i} \in \mathcal{S} \cup\{\emptyset\}$ for all $i$.

Proof. Let $y \in \mathbb{R}^{n} \backslash \bigcup_{S \in \mathcal{S}} S$. Let $\mathcal{B}$ contain $\mathbb{R}^{n}$ and all generalized balls with boundary in $\mathcal{S}$ and let $\mathcal{B}_{1}$ contain those of $\mathcal{B}$ that contain $y$. Since the generalized spheres from $\mathcal{S}$ are non-crossing, for every $B, B^{\prime} \in \mathcal{B}$ we have that $B \cap B^{\prime}=\emptyset$ or $B \subset B^{\prime}$ or $B^{\prime} \subset B$. According to Lemma 2 and using that $\mathcal{S}$ is closed and $\mathbb{R}^{n} \in \mathcal{B}_{1}$, Zorn lemma gives us a minimal generalized ball $B_{0}$ from $\mathcal{B}_{1}$. Let $\mathcal{B}_{2}$ be the family of those generalized balls $B \in \mathcal{B}$ for which $B \subset B_{0}$ and $y \notin B$. By Hausdorff maximal principle, each generalized ball from $\mathcal{B}_{2}$ is a subset of a maximal generalized ball from $\mathcal{B}_{2}$; these maximal balls are disjoint, hence there are only countably many of them and we may order them into a sequence $B_{1}, B_{2}, \ldots$. Clearly, any point which is not in $U=B_{0} \backslash \bigcup_{i \geq 1} \bar{B}_{i}$ is 
separated from $y$ by an $S \in \mathcal{S}$. On the other hand, every point of $B_{0}$ that is separated from $y$ belongs to some generalized ball from $\mathcal{B}_{2}$, hence to some $B_{k}$.

A set $K$ will be called a $K$-set about a generalized sphere $S$ if either $K=S$ or it is a non-empty set of the form $B_{0} \backslash \bigcup_{i \geq 1} \bar{B}_{i}$, where $B_{0}$ is a generalized (open) ball with radius at least $1 ; B_{1}, B_{2}, \ldots \subset B$ is a (finite or infinite) sequence of pairwise disjoint generalized balls with radii at least 1 ; and $\partial C_{i}=$ $S$ for an $i \geq 0$.

Lemma 5 showed that a non-empty closed family of pairwise non-crossing generalized spheres defines $K$-sets such that their union is $\mathbb{R}^{n}$ and the intersection of any two contains at most one point. As we will see later, any element of the Dynkin system generated by the large balls (in at least 3 dimension) can be built using these building blocks.

Lemma 6. Suppose that in $\mathbb{R}^{n}$, where $n \geq 3, K$ and $K^{\prime}$ are $K$-sets about crossing generalized spheres $S$ and $S^{\prime}$, respectively. Then $K \cap K^{\prime}$ is uncountable.

Proof. The boundary of a $K$-set about a generalized sphere $S$ is clearly always of the form $S \cup S_{1} \cup S_{2} \cup \ldots$, where $S, S_{1}, S_{2}, \ldots$ is a (finite or infinite) sequence of pairwise non-crossing generalized spheres with radii at least 1. For $K$ and $K^{\prime}$ this way we get $\partial K=S \cup S_{1} \cup S_{2} \cup \ldots$ and $\partial K^{\prime}=S^{\prime} \cup S_{1}^{\prime} \cup S_{2}^{\prime} \cup \ldots$

The intersection of the crossing generalized spheres $S$ and $S^{\prime}$ is a generalized sphere in an $(n-1)$-dimensional affine subspace, so (since $n-1 \geq 2)$ it is an uncountable set. Thus, by Lemma 4 , there must be a point $x \in S \cap S^{\prime}$ such that none of $S_{1}, S_{2}, \ldots, S_{1}^{\prime}, S_{2}^{\prime}, \ldots$ contains $x$. Since $S_{1}, S_{2}, \ldots, S_{1}^{\prime}, S_{2}^{\prime}, \ldots$ have radii at least 1 , a small neighborhood of $x$ is disjoint to $S_{1}, S_{2}, \ldots, S_{1}^{\prime}, S_{2}^{\prime}, \ldots$. Then in all cases it is easy to see that this neighborhood contains uncountable points of $K \cap K^{\prime}$.

We say that the class $\mathcal{S}$ of generalized spheres separates $E$ modulo $M$ if each generalized sphere of $\mathcal{S}$ and each equivalence class of $\sim_{\mathcal{S}}$ is contained in $E \cup M$ or $E^{c} \cup M$. The generalized sphere $A$ is essential for separating $E$ modulo $M$ if for every $x \in A \backslash M$ there exists arbitrarily close $y \in M^{c}$ such that $y \in E$ if $x \in E^{c}$ and $y \in E^{c}$ if $x \in E$.

Lemma 7. If in $\mathbb{R}^{n}$ (where $n \geq 3$ ), for $i=1,2$, the closed family of pairwise non-crossing generalized spheres $\mathcal{S}_{i}$ separates $E_{i}$ modulo $M_{i} ; A_{i} \in \mathcal{S}_{i}$ is essential for separating $E_{i}$ modulo a countable set $M_{i}$; and $A_{1}, A_{2}$ are crossing then $E_{1} \cap E_{2}$ is non-empty.

Proof. By Lemma 5 each equivalence class of $\sim_{\mathcal{S}_{i}}$ is a $K$-set. Since $\mathcal{S}_{i}$ separates $E_{i}$ modulo $M_{i}$ and $A_{i} \in \mathcal{S}_{i}$ is essential for separating $E_{i}$ modulo $M_{i}$, 
we have $K$-set $K_{i} \subset E_{i} \cup M$ about generalized sphere arbitrarily close to $A_{i}$. By Lemma 3 and Lemma 6 , this implies that $E_{1} \cap E_{2}$ is non-empty.

Lemma 8. For every set $E$ from the Dynkin system generated by the balls of $\mathbb{R}^{n}$ (for $n \geq 3$ ) with radii $\geq 1$ one may choose a countable set $M$ and a closed (in the metric d) family $\mathcal{S}$ of pairwise non-crossing generalized spheres such that $\mathcal{S}$ separates $E$ modulo $M$ and each generalized sphere of $\mathcal{S}$ is essential for separating $E$ modulo $M$.

Proof. If $E$ is a ball, it suffices to take $\mathcal{S}=\{\partial E\}$ and $M=\emptyset$. If the statement of the lemma holds for $E$ with some $M$ and $\mathcal{S}$, then it also holds for $E^{c}$ with the same $M$ and $\mathcal{S}$.

Hence we just have to find the required set $M$ and family $\mathcal{S}$ assuming that $E=\bigcup_{k=1}^{\infty} E_{k}$, the sets $E_{k}$ are disjoint, and there are countable sets $M_{k}$ and closed families $\mathcal{S}_{k}$ of pairwise non-crossing generalized spheres such that for each $k, \mathcal{S}_{k}$ separates $E_{k}$ modulo $M_{k}$ and each generalized sphere of $\mathcal{S}_{k}$ is essential for separating $E_{k}$ modulo $M_{k}$.

Define $\mathcal{S}^{\prime}$ as the family of those generalized spheres that belong to at least one of the $\mathcal{S}_{k}$ and $\mathcal{S}^{\prime \prime}$ as the closure of $\mathcal{S}^{\prime}$ (in the metric $d$ ). By Lemma $7, \mathcal{S}^{\prime}$ is a family of pairwise non-crossing generalized spheres; and by Lemma 2 and Lemma 3 , so is $\mathcal{S}^{\prime \prime}$. Let $M_{0}$ be the set of those points that belong to at least two different generalized spheres from $\mathcal{S}^{\prime \prime}$. By Lemma $4, M_{0}$ is countable, and so $M=\bigcup_{k=0}^{\infty} M_{k}$ is countable.

We claim that each generalized sphere $T \in \mathcal{S}^{\prime \prime}$ is contained in $E \cup M$ or $E^{c} \cup M$. Indeed, suppose to the contrary that $x \in(T \cap E) \backslash M$ and $y \in(T \backslash E) \backslash M$ and choose $k$ such that $x \in E_{k}$. Since $x \in E_{k} \backslash M_{k}, y \in E_{k}^{c} \backslash M_{k}$ and $\mathcal{S}_{k}$ separates $E_{k}$ modulo $M_{k}$, we get that $T \notin \mathcal{S}_{k}$. Since $x \notin M_{0}, x$ is not contained by any generalized sphere from $\mathcal{S}^{\prime \prime} \backslash\{T\} \supset \mathcal{S}_{k}$, so $x$ is contained in an equivalence class of $\sim_{\mathcal{S}_{k}}$. Using that $\mathcal{S}_{k}$ separates $E_{k}$ modulo $M_{k}$ we get that this equivalence class is in $E_{k} \cup M_{k}$, so $y$ is not in this equivalence class, which means that $x, y$ are separated by a generalized sphere $S \in \mathcal{S}_{k} \subset \mathcal{S}^{\prime \prime}$. But then $S, T \in \mathcal{S}^{\prime \prime}$ are crossing, which is a contradiction.

We prove that every $S \in \mathcal{S}^{\prime \prime}$ such that $S \cap\left(E^{c} \backslash M\right) \neq \emptyset$ is essential for separating $E$ modulo $M$ : The preceding paragraph shows that then $S \backslash M \subset$ $E^{c}$. Moreover, for every $\varepsilon>0$ we find $T \in \mathcal{S}_{k}$ such that $d(T, S)<\varepsilon / 2$ and, using that $T$ is essential for separating $E_{k}$ modulo $M_{k}$, for any $x \in S \backslash M \subset$ $E^{c} \subset E_{k}^{c}$ there exists arbitrarily close $y \in E_{k} \subset E$.

Finally, we define $\mathcal{S}$ as the family of those generalized spheres of $\mathcal{S}^{\prime \prime}$ that are essential for separating $E$ modulo $M$. Thus $\mathcal{S}$ is clearly a closed subset of $\mathcal{S}^{\prime \prime}$, so it remains to prove that each equivalence class of $\sim_{\mathcal{S}}$ is contained in either $E \cup M$ or in $E^{c} \cup M$. Let $x \in E \backslash\left(M \cup \cup_{S \in \mathcal{S}} S\right)$ and $y \in E^{c} \backslash\left(M \cup \bigcup_{S \in \mathcal{S}} S\right)$. 
We need to show that $x \chi_{\mathcal{S}} y$. If $S \cap\left(E^{c} \backslash M\right) \neq \emptyset$ for some $S \in \mathcal{S}^{\prime \prime}$ that separates $x$ and $y$, then (by the preceding paragraph) this $S$ belongs to $\mathcal{S}$, and we are done. We may therefore assume that $S \backslash M \subset E$ for every $S \in \mathcal{S}^{\prime \prime}$ that separates $x$ and $y$. By Lemma 5 , the equivalence class of $\sim_{\mathcal{S}^{\prime \prime}}$ that contains $y$ is of the form $G=B_{0} \backslash \bigcup_{i>1} \bar{B}_{i}$, where $B_{0}$ is a generalized (open) ball; $B_{1}, B_{2}, \ldots \subset B$ is a (finite or infinite) sequence of pairwise disjoint generalized balls; and $\partial B_{i} \in \mathcal{S}^{\prime \prime} \cup\{\emptyset\}$ for all $i$. Since each $\mathcal{S}_{k}$ separates $E_{k}$ modulo $M_{k}$, the class $\mathcal{S}^{\prime \prime}$ separates $E$ modulo $M$, so $x$ cannot be in $G$. It cannot be on the boundary of $G$ either, so $x, y$ are separated by one of the generalized spheres $\partial B_{i} \in \mathcal{S}^{\prime \prime}$. Then $\partial B_{i} \subset E \cup M$ but $G \subset E^{c} \cup M$, so $\partial B_{i}$ is essential for separating $E$ modulo $M$, hence it is in $\mathcal{S}$, which implies that $x \chi_{\mathcal{S}} y$.

The following result is clearly stronger than Theorem 1, which we wanted to prove:

Theorem 9. Every set $E$ of the Dynkin system generated by the balls of $\mathbb{R}^{n}$ $(n \geq 3)$ with radii $\geq 1$ has diameter at least 2 .

Proof. Using Lemma 8 and Lemma 5, up to some changes on a countable set, $E$ is the union of $K$-sets. On the other hand, clearly all $K$-sets have diameter at least 2 , even if we remove a countable set of points.

Remark 10. Lemma 8 is not true in 2-dimension: Let $F_{1}$ and $F_{2}$ be the union of the closed discs with radii 1 and with centers of the form $(2 i, 2 j)$ and $(2 i+1,2 j+1)$, respectively. Then $E=F_{1}^{c} \cup F_{2}^{c}$ is a counter-example.

The author does not know weather Theorem 9 (or at least Theorem 1) holds in 2-dimension.

In the remaining part of this note we give a complete characterization of the Dynkin system generated by the large balls of $\mathbb{R}^{n}$ for $n \geq 3$. Some of the details of the proof will be omitted.

Theorem 11. For $n \geq 3$ a set $E \subset \mathbb{R}^{n}$ is in the Dynkin $\mathcal{D}$ system generated by the balls of $\mathbb{R}^{n}$ with radii $\geq 1$ if and only if

(1) E is a Borel set, and

(2) there is a collection $\mathcal{K}$ of $K$-sets such that any two of them have at most one common point and the symmetric difference of $\cup \mathcal{K}$ and $E$ is countable.

Proof. If $E$ is in the Dynkin system then (1) is obvious; Lemma 8 and Lemma 5 imply (2).

For the other direction first note that any one point set is in the Dynkin system $\mathcal{D}$. (Indeed, using that $\mathcal{D}$ is clearly also closed under monotone union 
and monotone intersection and contains all the large closed balls as well, one can first generate the union of a large open ball and a point of its boundary, then the union of the complement of this set and the open ball is the complement of the point.) Thus, if we modify a set of $\mathcal{D}$ on a countable set, the set we get is still in $\mathcal{D}$. Therefore we can assume that $E=\cup \mathcal{K}$. Moreover, for generating the Dynkin system, we can also allow pairwise "almost" disjoint countable union as well, where by almost disjoint we mean that the intersection is a countable set.

Let $\mathcal{K}_{1}$ contain those $K$-sets of $\mathcal{K}$ that are generalized spheres and let $\mathcal{K}_{2}$ contain the others. Since $\mathcal{K}_{2}$ contains disjoint open sets, it is countable. On the other hand, each $K$-set is obviously in the Dynkin system $\mathcal{D}$, so we get that $\cup \mathcal{K}_{2} \in \mathcal{D}$. Using that $\mathcal{K}$ contains pairwise almost disjoint $K$-sets, $\mathcal{K}_{1}$ contains generalized spheres and $\mathcal{K}_{2}$ contains open sets, we deduce that $\cup \mathcal{K}_{1}$ and $\cup \mathcal{K}_{2}$ are disjoint. Thus $\cup \mathcal{K}_{1}$ is a Borel set and we need to prove that it is also in $\mathcal{D}$.

Let $\mathcal{B}$ be the class of all generalized balls with boundary in $\mathcal{K}_{1}$. Since $\mathcal{K}_{1}$ is a class of pairwise non-crossing spheres, for any $B_{1}, B_{2} \in \mathcal{B}$ we have either $B_{1} \cap B_{2}=\emptyset$ or $B_{1} \subset B_{2}$ or $B_{2} \subset B_{1}$. Using this property, the following relation is an equivalent relation on $\mathcal{B}: B_{1} \sim B_{2}$ if they are contained in each other and whenever $B \in \mathcal{B}$ meets one of them it also meets the other. Using that all balls from $\mathcal{B}$ have radii at least 1 , one can check that the set of equivalence classes is countable: $\mathcal{B}_{1}, \mathcal{B}_{2}, \ldots$. Let $\mathcal{C}_{1}, \mathcal{C}_{2}, \ldots$ be the corresponding classes of generalized spheres from $\mathcal{K}$. Since $\cup \mathcal{C}_{1}, \cup \mathcal{C}_{2}, \ldots$ are almost disjoint it is enough to prove that each of them (say $\cup \mathcal{C}_{1}$ ) is in the Dynkin system. The set $\cup \mathcal{C}_{1}$ is Borel since it is, up to a countable set, the intersection of the Borel set $\cup \mathcal{K}$ and a closed set of the form $\overline{B_{1}} \backslash B_{2}$, where $B_{2} \subset B_{1}$ are generalized balls.

It is easy to extend $\mathcal{C}_{1}$ to a class $\mathcal{C} \supset \mathcal{C}_{1}$ of pairwise non-crossing generalized spheres with radii at least 1 such that they cover the whole $\mathbb{R}^{n}$ except a ball $B(O, 1)$ with radius 1 . By Lemma 4 every point is covered once except a countable set. Let $e$ be a line through $O$ that avoid all these exceptional points. Let $f \subset e$ be the closed half-line that has distance 1 from $O$ and let $F=\left(\cup \mathcal{C}_{1}\right) \cap f$. Then $\Psi(S)=S \cap f$ is a bijection between the generalized spheres of $\mathcal{C}$ and the points of $f$. Clearly $\Psi\left(\cup \mathcal{C}_{1}\right)=F$ and $F$ is a Borel subset of $f$. On the other hand, in a half-line the Dynkin system generated by the subintervals obviously contains all Borel sets. The pre-image $\left(\Psi^{-1}\right)$ of a subinterval is of the form $B_{1} \backslash \overline{B_{2}}\left(B_{1} \supset B_{2} \in \mathcal{C}\right)$, so it is clearly in the Dynkin system $\mathcal{D}$ generated by the large balls. The same way as we can generate $F$ from the subintervals of $f$ we can generate $\cup \mathcal{C}_{1}$ from the corresponding sets of the form $B_{1} \backslash \overline{B_{2}}$, so $\cup \mathcal{C}_{1} \in \mathcal{D}$.

Acknowledgment. The author would like to thank Professor David 
Preiss for the helpful discussions.

\section{References}

[1] Jackson, S.; Mauldin, R. D., On the $\sigma$-class generated by open balls, Proc. Cambridge Phil. Soc., to appear.

[2] Keleti, T.; Preiss, D., The balls do not generate all Borel sets using complements and countable disjoint unions, Proc. Cambridge Phil. Soc., to appear.

[3] Mejlbro, L.; Preiss, D.; Tišer, J., Positivity Principles in Geometrical Measure Theory, Proc. of the Measure Theory Conference, Oberwolfach, 1990, Supplemento ai Rendiconti del Circolo Matematico di Palermo, Serie II - numero 28 - anno 1992, 163-167.

[4] Mejlbro, L.; Preiss, D.; Tišer, J., Determination and differentiation of measures, forthcoming book.

[5] Olejček, V., Generation of a q- $\sigma$-algebra in the plane, Proc. Conf. Topology and Measure V, Greifswald, 1988, 121-125.

[6] Olejček, V., The $\sigma$-class generated by balls contains all Borel sets, Proc. Amer. Math. Soc. 123 (1995), 3665-3675.

[7] Preiss, D., Differentiation of measures in infinitely dimensional spaces, Proc. Conf. Topology and Measure III, Greifswald, 1982, 201-207.

[8] Zelený, M., The Dynkin system generated by balls in $\mathbb{R}^{d}$ contains all Borel sets, submitted. 